\title{
Reliability Modeling and Optimization Using Fuzzy Logic and Chaos Theory
}

\author{
Alexander Rotshtein, ${ }^{1}$ Denys Katielnikov, ${ }^{2}$ and Ludmila Pustylnik ${ }^{3}$ \\ ${ }^{1}$ Department of Industrial Engineering and Management, Jerusalem College of Technology-Machon Lev, Jerusalem 91160, Israel \\ ${ }^{2}$ Department of Information Technology and Computer Engineering, Vinnytsia National Technical University, \\ Vinnitsia 21021, Ukraine \\ ${ }^{3}$ Department of Mechanical Engineering, Afeka-Tel Aviv Academic College of Engineering, Tel Aviv 69107, Israel
}

Correspondence should be addressed to Alexander Rotshtein, rot@jct.ac.il

Received 6 February 2012; Accepted 3 September 2012

Academic Editor: Satish Bukkapatnam

Copyright (C) 2012 Alexander Rotshtein et al. This is an open access article distributed under the Creative Commons Attribution License, which permits unrestricted use, distribution, and reproduction in any medium, provided the original work is properly cited.

Fuzzy sets membership functions integrated with logistic map as the chaos generator were used to create reliability bifurcations diagrams of the system with redundancy of the components. This paper shows that increasing in the number of redundant components results in a postponement of the moment of the first bifurcation which is considered as most contributing to the loss of the reliability. The increasing of redundancy also provides the shrinkage of the oscillation orbit of the level of the system's membership to reliable state. The paper includes the problem statement of redundancy optimization under conditions of chaotic behavior of influencing parameters and genetic algorithm of this problem solving. The paper shows the possibility of chaos-tolerant systems design with the required level of reliability.

\section{Introduction}

The classical reliability theory $[1,2]$ is based on the probabilistic approach. Essential limitations of this approach are connected with "the problem of the source data" which depend on many factors and which may not correspond to the real conditions of the system's functioning. Besides, the statistical data used in the probabilistic reliability models fix only the facts of real failures and do not contain the information about the causes of these failures. Whereas the causes of these failures are connected with the elements' variables (temperature, humidity, tension, etc.), which become more (or less) than a certain critical level. So, we can affirm that the probabilistic theory $[1,2]$ models the reliability in the space of events effects (i.e., failures) and suits badly for the reliability modelling in the space of events causes (i.e., variables).

The alternative for the probabilistic modeling of the reliability is the approach based on the fuzzy logic [3] and related possibility theory [4]. In this case the classical "failure probability" is replaced by "failure possibility" which is modeled by the membership function of the system (or the element) variables to the reliable state (Figure 1).

The explicit dependence of the membership function on the variables (failure causes) makes convenient the integration of the fuzzy model of reliability with the technique of time series [5], which allows observing the change of the reliability level in the real time.

The chaos theory is a new approach to the analysis of nonlinear time series [6]. It uses the conceptual apparatus of the theory of nonlinear oscillations [7] and purposes to study the phase portrait of the dynamical system with its intrinsic states of stability (attractors) and bifurcations, that is, "jumps" between stable states. Unlike the classical oscillation theory [7] where the phase portrait is formed on the base of the system description by means of differential equations, the chaos theory [6] offers the methods of the phase-portrait extraction from the experimental data, that is, directly from the time series.

The integration of fuzzy reliability model with the phase portraits of variables creates preconditions for the construction of phase portrait reflecting the system reliability 


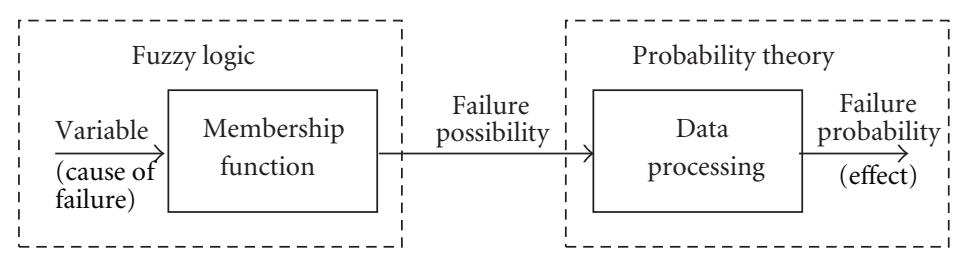

FIGURE 1: Relationship of the probability theory and fuzzy logic in reliability estimation.

dynamics. The pattern of bifurcations which may be interpreted as failure instants is of particular interest.

The works on the fuzzy reliability theory began in 90 s of the last century. The first specialized collection of papers in this field is the edited volume [8]. The first monographs containing the approaches to the construction of fuzzy reliability theory are [9-11]. In the works $[12,13]$ there is a fuzzy algorithmic approach to reliability modeling based on the algebra of regular algorithms [14] and expert assessments of the performance correctness of operators and conditions by means of membership functions [3]. The problem of reliability optimization of the control resources based on fuzzy algorithmic approach is solved in [15].

An approach to the online reliability evaluation based on the integration of fuzzy logic and forecasting methods of time series (exponential smoothing and Kalman filtering) is proposed in [16].

The idea of chaos theory application in the reliability modeling appears in [17]. Two real data bases about software failures are processed by the methods of chaos theory in the paper [18]. It was shown that the deterministic model of failures is more adequate to the experimental data than the traditional stochastic models, for example, the modified Poisson's law, and so forth. The results of the work [18] can be considered as a new approach (alternative to the statistical) to the data processing about the failures on the level of elements. We do not know any publications about the chaos theory applications to the reliability modeling of the system taking into account its structure.

In this connection there are following the questions.

(1) How does the system structure influence the phase portrait of reliability?

(2) Is it possible to solve the redundancy optimization problem with deterministic (chaotic) order of the occurrences of failures?

As far as the failures are connected with the oscillations of variables, then the answers for these questions should be searched on the base of integration of the fuzzy logic and chaos theory.

In this paper we use the fuzzy algorithmic approach to parametric reliability modeling proposed in $[12,13]$, and the simple generator of chaotic oscillations of variables in the form of logistic function [19]. The further description is organized in the following way.

Section 2 describes the principles of reliability dynamics modelling by means of the composition of membership function and a generator of chaos.
Section 3 examines the fuzzy reliability model of an element with a multiple redundancy. We consider the results of computer experiment on the analysis of reliability level bifurcations depending on the multiplicity of redundancy.

Section 4 considers the redundancy optimization problem under the conditions of chaotic oscillations of the parameters of the elements of systems.

\section{Basic Principles}

The fuzzy chaotic approach to the reliability dynamics modeling is based on the following principles.

2.1. Fuzzy Correctness. According to this principle introduced in $[12,13]$, there is not a crisp boundary between "correct" (1) and "incorrect" (0) results of the functioning of a system and its elements. For the formal evaluation of the correctness level it is used the multidimensional (by the number of variables) membership function $\mu^{1}\left(x_{1}, x_{2}, \ldots\right)$ which depends on the measured parameters (input variables). The correctness of each variable is determined by the membership function $\mu^{1}\left(x_{i}\right)$ of the variable $x_{i}$ to the correct value.

The function $\mu^{1}\left(x_{i}\right)$ can be interpreted as the correctness distribution of the variable $x_{i}$ : extreme cases $\mu^{1}\left(x_{i}\right)=1(0)$ correspond to the maximal (minimal) level of the correctness of the variable $x_{i}$. Pay attention that the correctness distribution $\mu^{1}\left(x_{i}\right)$ satisfies the axioms of fuzzy sets theory [3], in contrast to the probabilistic distributions used in the classical reliability theory $[1,2]$.

The typical correctness distributions (membership functions) are represented in Figure 2. They correspond to three possible cases of fuzzy boundaries between "correct" (1) and "incorrect" (0):

(a) correct (1)-incorrect (0),

(b) incorrect (0)—correct (1)—incorrect (0),

(c) incorrect (0) correct (1).

2.2. Integration of Membership Functions and Time Series. It is assumed that for the variable $x$ it is known the time series of its values $\left(x_{1}, x_{2}, x_{3}, \ldots\right)$ in discrete moments of time $\left(t_{1}, t_{2}, t_{3}, \ldots\right)$. Putting these values in the membership function $\mu^{1}(x)$, we receive the dynamics of the correctness level of the variable $x$ in the form of the function $\mu^{1}[x(t)]$, Figure 3. 


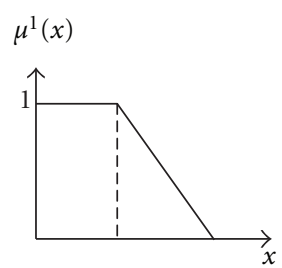

(a)

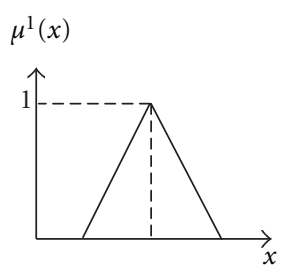

(b)

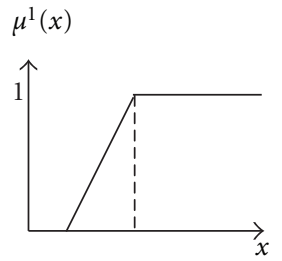

(c)

FIGURE 2: The typical correctness distributions.

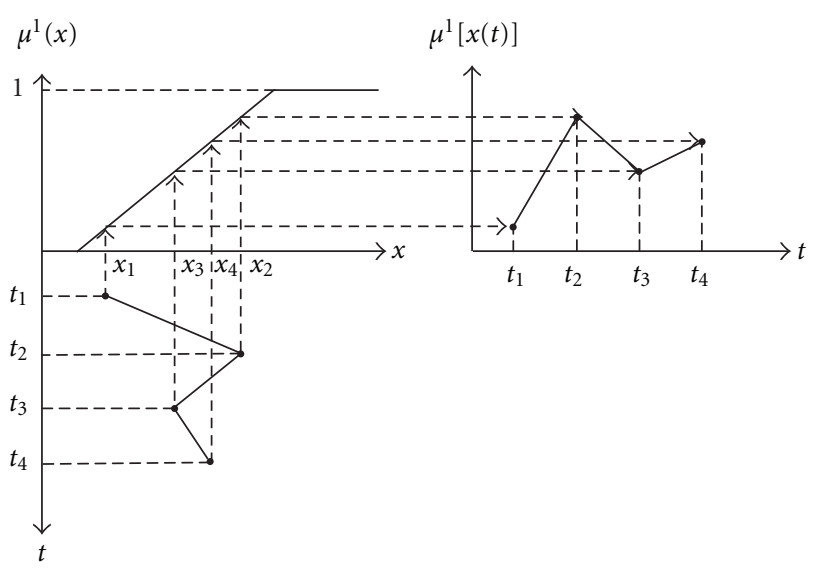

FIGURE 3: Integration of membership functions and time series.

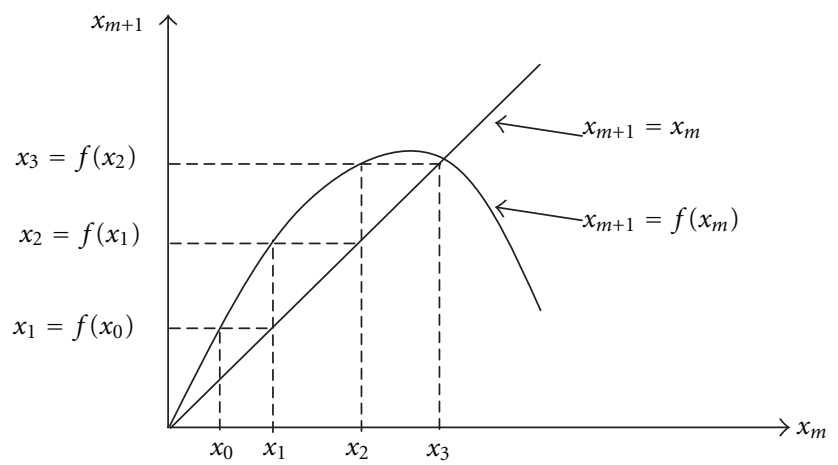

FIGURE 4: Iterative Lamerey diagram.

2.3. Chaos Generator. Chaos means the oscillations which seem random but in truth they are generated by the deterministic nonlinear model. In [6] about 40 models of the chaos generators are described. Each model contains variables whose values must be fitted on the base of the experimental data. Algorithm of the chaos generation is explained by means of iterative Lamerey diagram, widely used in the classical theory of nonlinear oscillations [7].

It is assumed that there is a known function $x_{m+1}=$ $f\left(x_{m}\right)$, connecting two neighboring elements of time series: $x_{m}$ and $x_{m+1}$. Iterative diagram consists of this function and the bisector $x_{m}=x_{m+1}$ (Figure 4$)$. Choosing the initial point $x_{0}$ by means of vertical and horizontal lines we obtain the points on the axis $x_{i}$ as follows:

$$
x_{1}=f\left(x_{0}\right), \quad x_{2}=f\left(x_{1}\right), \quad x_{3}=f\left(x_{2}\right), \ldots
$$

The most popular generator of chaos is given by the logic map [19] as follows:

$$
x_{m+1}=f\left(x_{m}\right)=a x_{m}\left(1-x_{m}\right), \quad 0 \leq x_{m} \leq 1,
$$

where $0 \leq a \leq 4$ is the control parameter determining the nature of chaotic orbits.

Using the iterative equation (2) we can generate the consequence $x_{1}, x_{2}, \ldots$ for the given parameter $a$ and initial point $x_{0}$.

For example, if $x_{0}=0.25$ and $a=0.5$, then we find that

$x_{1}=0.094, x_{2}=0.042, x_{3}=0.020, x_{4}=0.010, x_{5}=$ $0.005, \quad x_{6}=0.002, x_{7}=0.001, \quad x_{8}=0.001, \quad x_{9}=$ 0.000 .

If $x_{0}=0.25$ and $a=2.0$, then

$x_{1}=0.375, x_{2}=0.469, x_{3}=0.498, x_{4}=x_{5}=x_{6}=$ $\cdots=0.5$.

With the corresponding values of the parameter $a$ it is possible to get different types of attractors (Figure 5) by means of iteration algorithm (Figure 4):

(a) stable focus $(a=2.8)$, (b) stable orbit $(a=3.2)$, (c) double orbit $(a=3.5)$, and (d) chaotic orbit $(a=3.6)$.

Increasing gradually the parameter $a$, it is possible to observe the moments of bifurcations, that is, transitions from one type of the attractor to another. Figure 6 shows that in the moment $a=3$ there is a jump from one stable state to two other stable states. In the moment $a=3.449 \ldots$ the number of stable states is doubled, and so forth. The moment $a=4$ corresponds to the complete chaos [19]. The described chaos generator will be used further for the integration with the fuzzy reliability model.

\section{Fuzzy Chaotic Reliability Model}

We consider the simple system with redundancy which is modeled by fuzzy algorithmic approach proposed in [12, 13] and logistic function (2). 


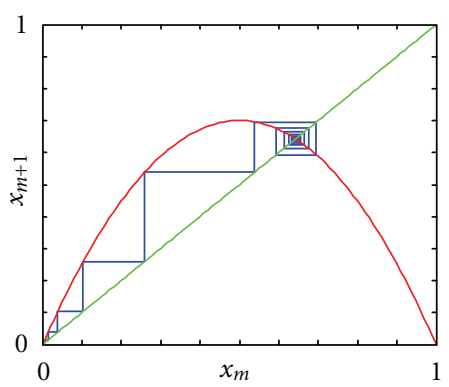

(a)

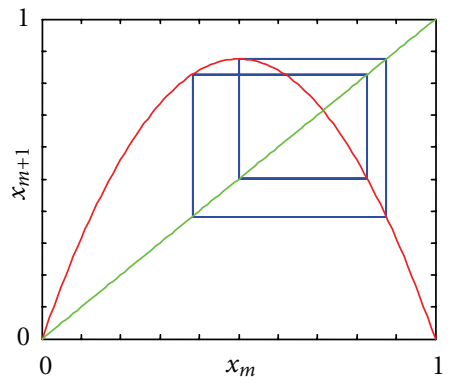

(c)

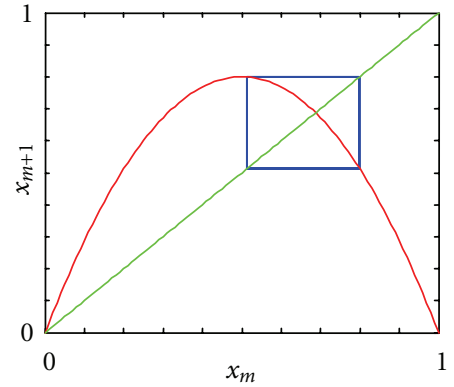

(b)

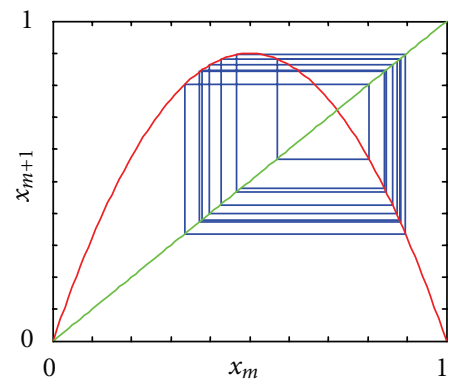

(d)

FIGURE 5: Different types of logistic map attractors: (a) stable focus, (b) stable orbit, (c) double orbit, and (d) chaotic orbit.

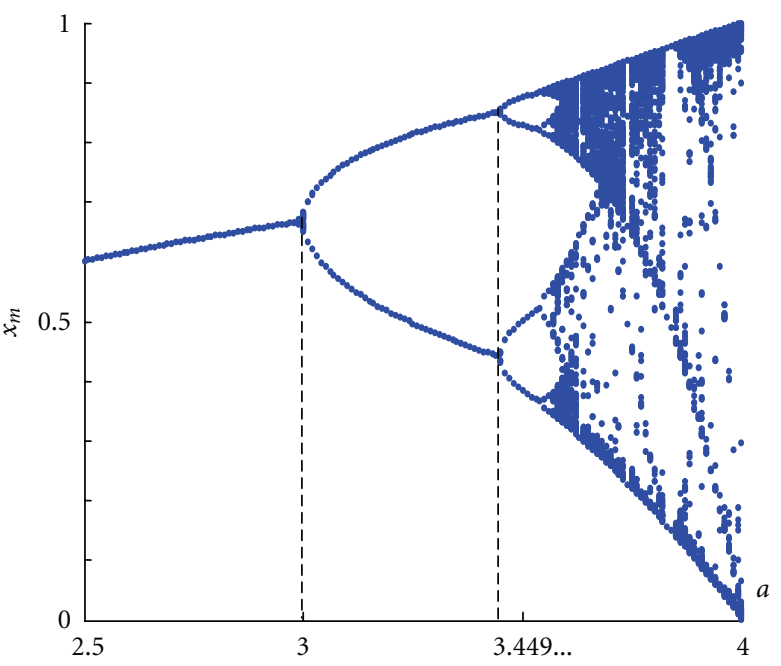

FIGURE 6: Bifurcation diagram of logistic map.

3.1. Element with Redundancy. The element with redundancy is presented in Figure 7 in the form of the parallel circuit where the primary element $(A)$ has $y$ of redundant elements $(y=0,1,2, \ldots)$. All the elements are supposed to be homogeneous.

The quality of the element $A$ functioning depends on the variable $x$ which varies during the time: $x=x(t)$. To evaluate the reliability of the element $A$ it is used: $\mu_{A}^{1}(x)$ is a membership function which determines correctness distribution of variable $x$ during the functioning of $A$.

The parallel circuit (Figure 7) assumes that the failure of the system requires the failure of all $(y+1)$ elements

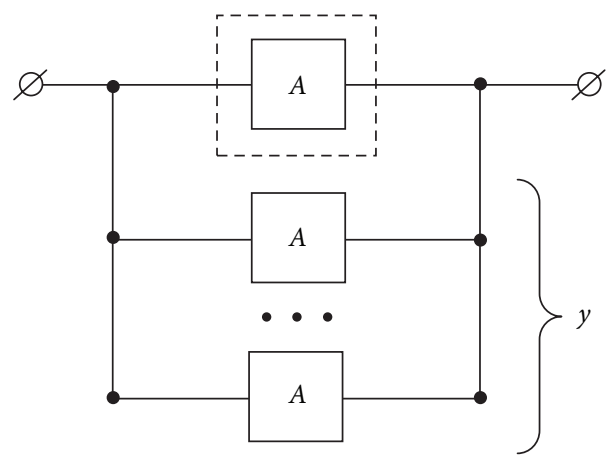

FIGURE 7: Element with redundancy.

similar to $A$. That is why the correctness of the element with redundancy functioning is evaluated by the following formula:

$$
\mu_{B}^{1}(y)=1-\left[1-\mu_{A}^{1}(x)\right]^{y+1}
$$

3.2. Reliability Bifurcation. The model (3) allows observing the dynamics of the system reliability level, that is, of the correctness function $\mu_{B}^{1}(y)$ during the chaotic oscillations of the variable $x$ according to the logistic map (2).

The purpose of the computer experiment consisted of the research of bifurcations of the correctness level $\mu_{B}^{1}(y)$ with different correctness distributions of the element $A$ and different redundancy rates $(y=1,3,5,7)$.

The experiment is carried out with two correctness distributions shown in Figure 8: triangle (a) and threshold (b). During the chaos generation the parameter $a$ of the 


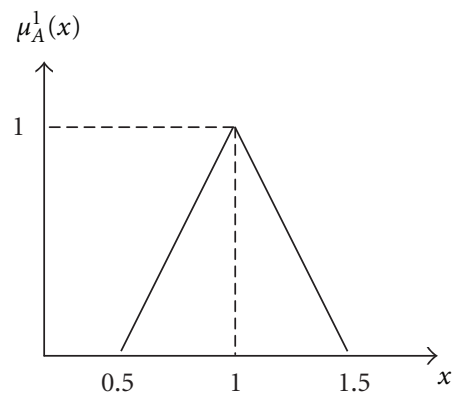

(a)

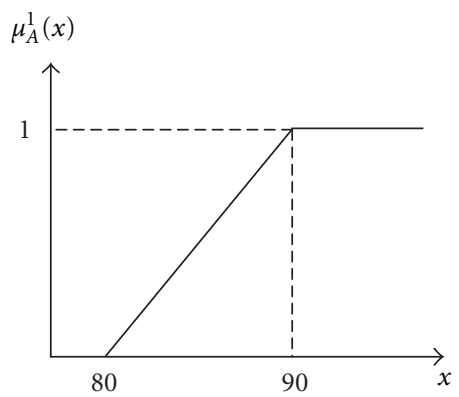

(b)

Figure 8: Correctness distributions of variable $x$ in the experiment.

logistic map (2) was changed in the range from 2.5 to 4 . For each distribution (Figure 8) we obtained 4 bifurcation diagrams, each of them corresponds to different redundancy rates $(y)$. The results are represented in Figures 9 and 10, where the horizontal axis is the chaos parameter $(a)$, and vertical axis is the reliability level $\mu_{B}^{1}(y)$.

Figures 9 and 10 show that in spite of the chaos growth (parameter $a$ ) by the increasing of redundancy rate $(y)$, it is possible

(a) to postpone the moment of the first bifurcation which is associated with the reliability loss and

(b) to decrease the diameter of an orbit around which there are oscillations of the level of system's membership to the stable state.

That is why we can consider a redundancy optimization problem under chaotic oscillations of the parameters of elements.

\section{Redundancy Optimization under Chaos of Parameters}

We consider a sequential system where each element has some level of redundancy. This system is described by the series-parallel structure (Figure 11), where $A_{i}$ is a component which depends on the variable $x_{i}, y_{i}$ is the redundancy rate of the component $A_{i}$, and $\mathbf{Y}=\left(y_{1}, y_{2}, \ldots, y_{n}\right)$ is the vector of the redundancy rates $y_{i}, i=1,2, \ldots, n$.

It is supposed to be known that

$\mu_{A_{i}}^{1}\left(x_{i}\right)$ is correctness distribution of variable $x_{i}$ during the element $A_{i}$ functioning,
TABLE 1: Correctness distribution of elements.

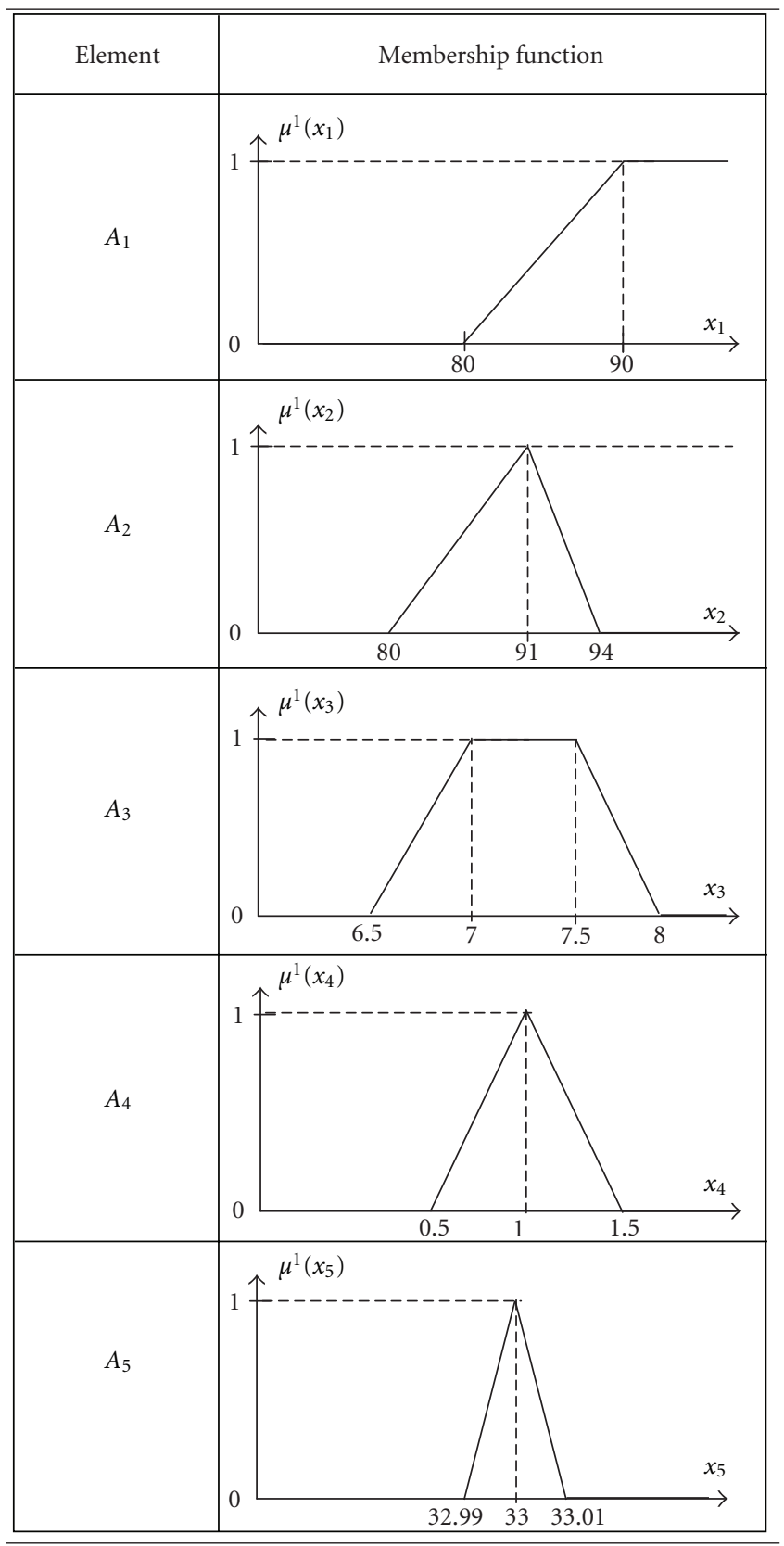

$\left[x_{i}, \overline{x_{i}}\right]$ is the range of possible values of the variable $x_{i}$ and

$c_{i}$ is mean cost of one redundant component like $A_{i}$.

For the system in the Figure 11 taking into account (3) we have

$$
\begin{gathered}
\mu_{B}^{1}(\mathbf{Y})=\prod_{i=1}^{n}\left\{1-\left[1-\mu_{A_{i}}^{1}\left(x_{i}\right)\right]^{y_{i}+1}\right\} \\
C_{B}(\mathbf{Y})=\sum_{i=1}^{n} c_{i} y_{i}
\end{gathered}
$$




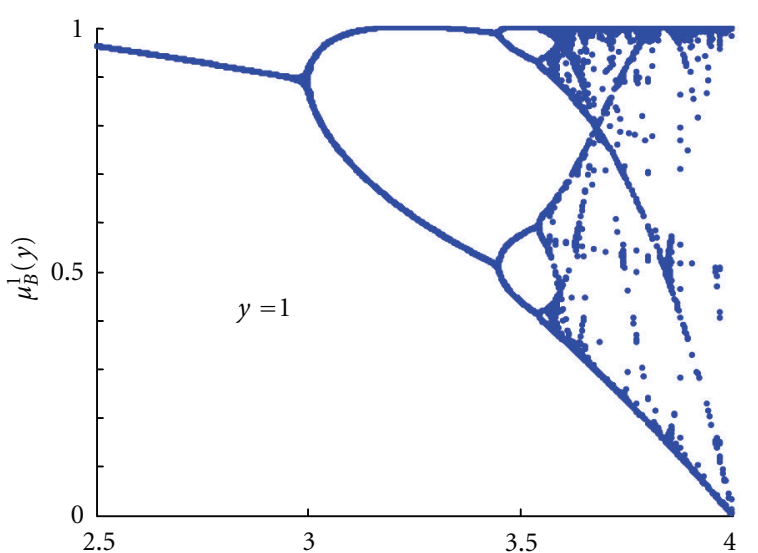

(a)

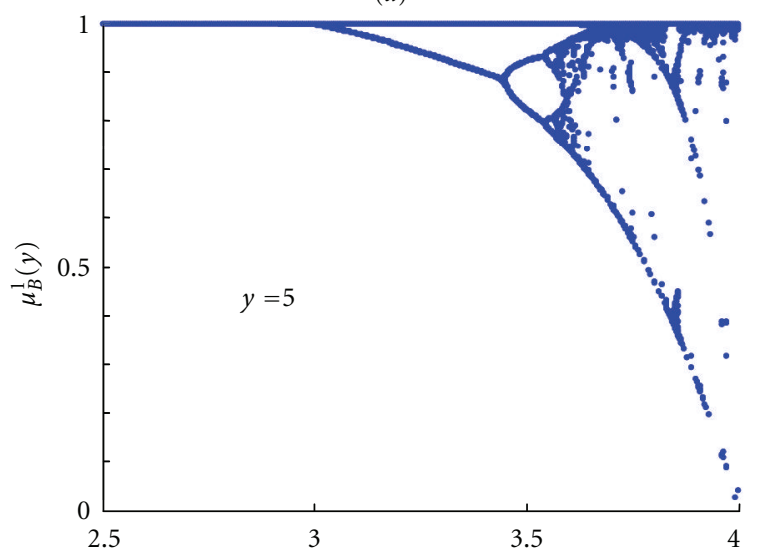

(a)

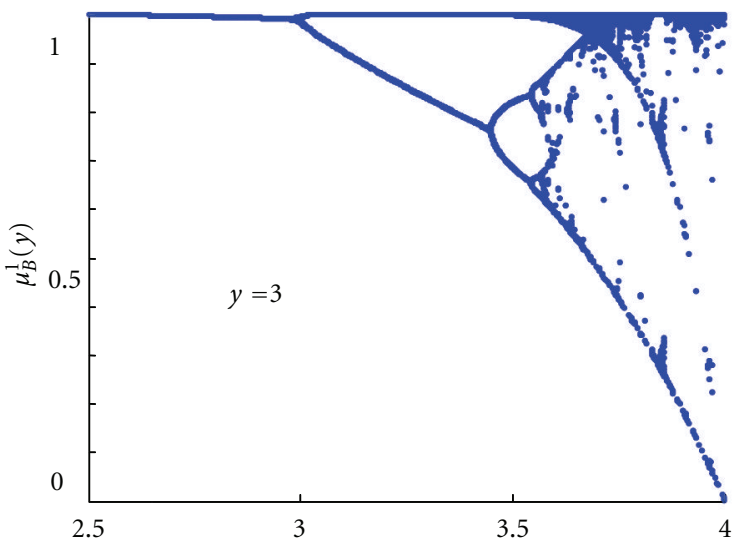

(a)

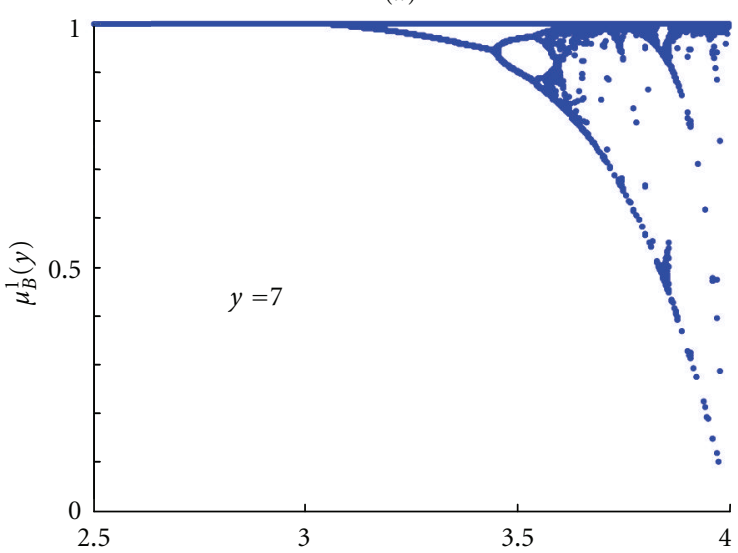

(a)

FIGURE 9: Reliability bifurcations for triangle correctness distribution.

where $\mu_{B}^{1}(\mathbf{Y})$ is the correctness of system's functioning with redundancy rates given by vector $\mathbf{Y}$, and $C_{B}(\mathbf{Y})$ is the total expenses for the redundancy.

It is supposed that the variable $x_{i}$ during the functioning of the component $A_{i}$ makes the chaotic oscillations according to the law (2) as follows:

$$
x_{i}(m+1)=a x_{i}(m)\left[1-x_{i}(m)\right],
$$

where $x_{i}(m)$ is the value of the variable $x_{i}$ on the $m$ th step of the logistic map.

One of the practically important problems of the redundancy optimization under chaotic oscillation of parameters can be formulated as follows:

to find the vector $\mathbf{Y}=\left(y_{1}, y_{2}, \ldots, y_{n}\right)$ which provides

$$
C_{B}(\mathbf{Y}) \longrightarrow \min ,
$$

subject to

$$
\mu_{B}^{1}(\mathbf{Y}) \in\left[\mu_{B}^{*}, 1\right], \quad a=a^{*},
$$

where $\mu_{B}^{*}$ is the minimal acceptable level of the correctness of system's functioning with redundancy of the elements.

$a^{*}$ is the parameter of the logistic map (6) determining the level of chaos.
TABle 2: Optimal redundancy rates $\left(\mu_{B}^{*}=0.8\right)$.

\begin{tabular}{lccccccc}
\hline \multirow{2}{*}{ Chaos parameter $\left(a^{*}\right)$} & \multicolumn{9}{c}{$Y$} & \multicolumn{3}{c}{ Criteria } \\
& $y_{1}$ & $y_{2}$ & $y_{3}$ & $y_{4}$ & $y_{5}$ & $C_{B}(Y)$ & $\mu_{B}(Y)$ \\
\hline 2.9 & 2 & 1 & 0 & 1 & 2 & 6 & 0.8167 \\
3.2 & 5 & 4 & 4 & 5 & 3 & 21 & 0.8065 \\
3.5 & 5 & 5 & 7 & 9 & 9 & 35 & 0.8043 \\
3.6 & 6 & 5 & 8 & 13 & 13 & 45 & 0.8027 \\
\hline
\end{tabular}

The problem was solved for $n=5, c_{i}=1, i=1,2, \ldots, 5$ and the correctness distributions from the Table 1. It was supposed that the variables $x_{i}$ for all the components $A_{i}$ have the same level of chaos (parameter $a^{*}$ ).

In order to solve the problem of optimization we used the genetic algorithm from the MATLAB presented in the Appendix.

Some of the optimal solutions are represented in Tables 2 and 3 for two minimum acceptable levels of the system functioning correctness ( 0.8 and 0.9 ) and four levels of chaos $\left(a^{*}\right)$.

Tables 2 and 3 show that by increasing redundancy rate under conditions of chaotic oscillations of parameters of elements it is possible to achieve the stabilization of system functioning correctness level in the required interval $\left[\mu_{B}^{*}, 1\right]$. 


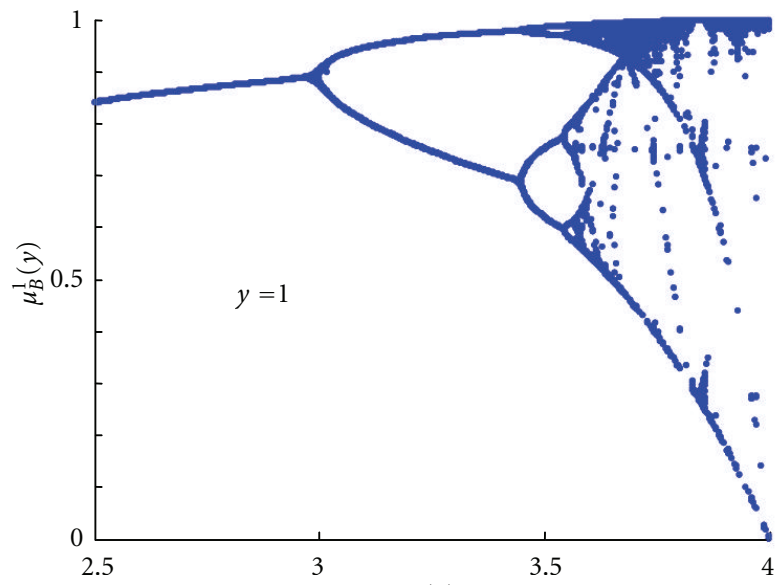

(a)

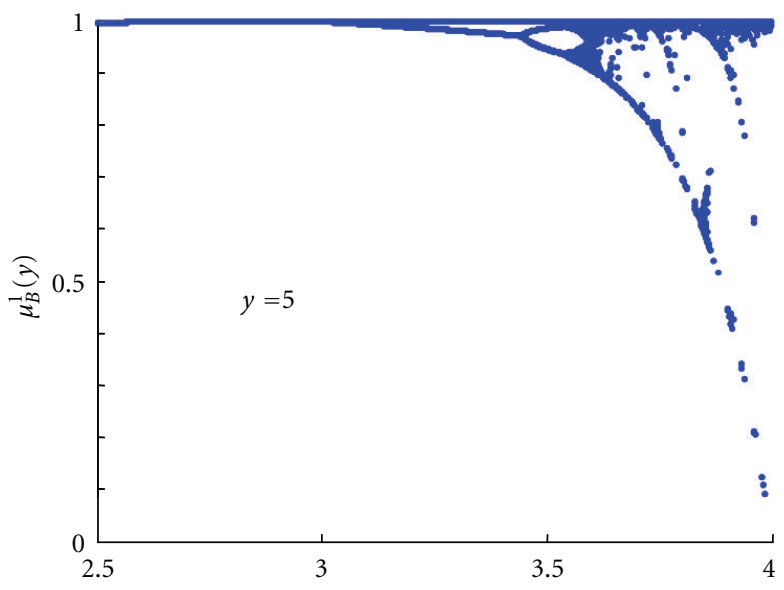

(a)

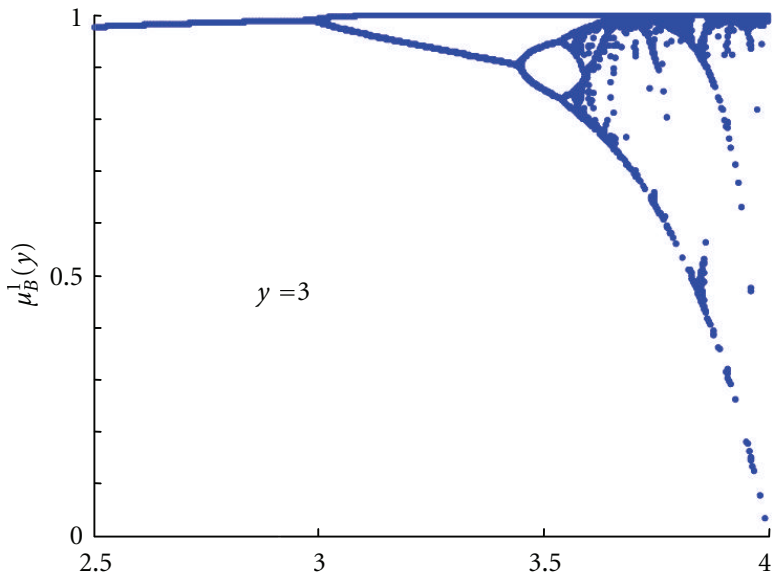

(a)

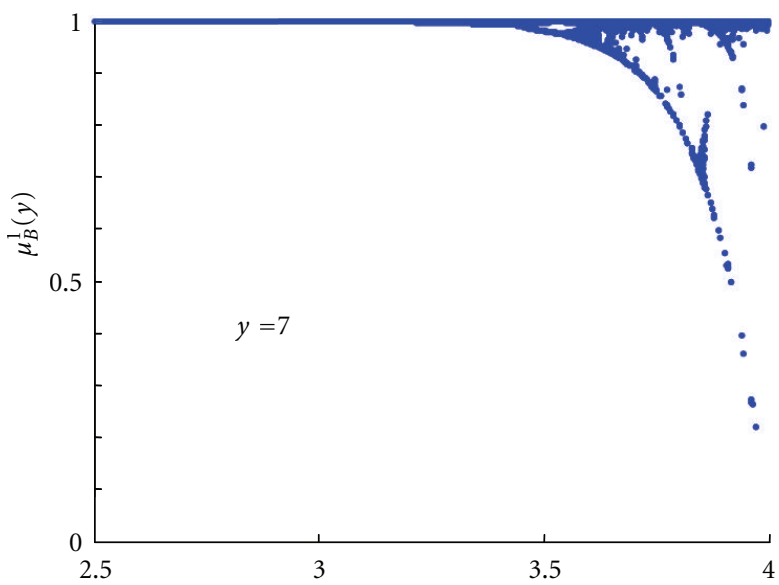

(a)

FIGURE 10: Reliability bifurcations for threshold correctness distribution.

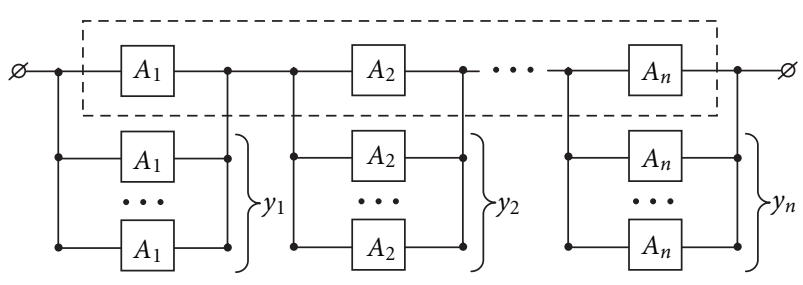

FIGURE 11: Sequential system with redundancy of elements.

TABLE 3: Optimal redundancy rates $\left(\mu_{B}^{*}=0.9\right)$.

\begin{tabular}{lccccccc}
\hline \multirow{2}{*}{ Chaos parameter $\left(a^{*}\right)$} & \multicolumn{3}{c}{$Y$} & & \multicolumn{2}{c}{ Criteria } \\
& $y_{1}$ & $y_{2}$ & $y_{3}$ & $y_{4}$ & $y_{5}$ & $C_{B}(Y)$ & $\mu_{B}(Y)$ \\
\hline 2.9 & 3 & 1 & 0 & 2 & 2 & 8 & 0.9017 \\
3.2 & 4 & 4 & 3 & 6 & 7 & 24 & 0.9015 \\
3.5 & 8 & 6 & 7 & 12 & 11 & 44 & 0.9006 \\
3.6 & 8 & 8 & 13 & 13 & 16 & 58 & 0.9002 \\
\hline
\end{tabular}

In the worst case system design the chaos parameter $a^{*}$ can be chosen as the biggest.

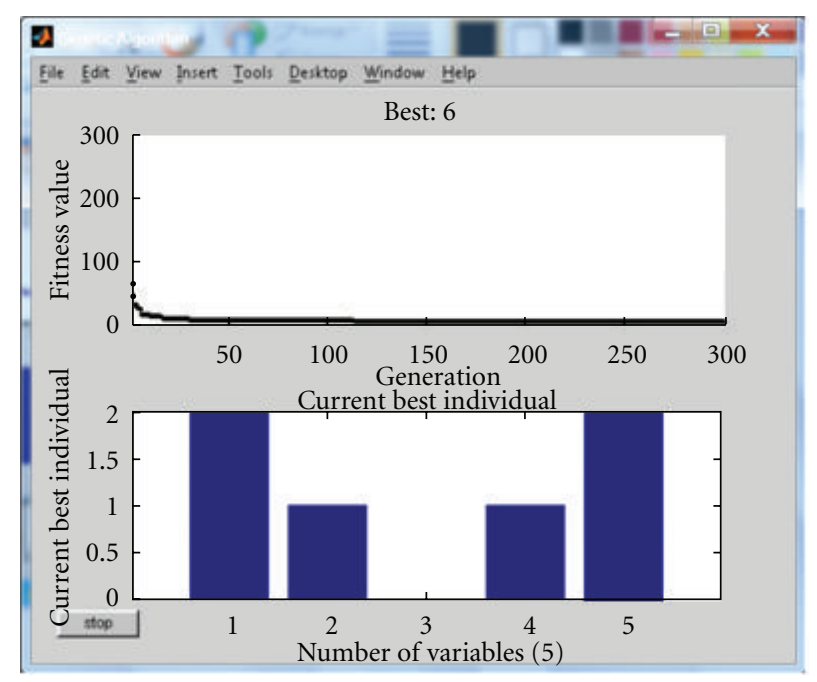

FIGURE 12: Solution found with genetic algorithm.

It is important to mention that the classical redundancy optimization problem [20] was solved without any statistic 
data about the reliability of elements. Instead of such a data we used expert information about fuzzy set membership functions describing the correctness distributions of parameters which influence the reliability.

\section{Conclusion}

The integration of fuzzy logic and chaos theory is a convenient methodology of the system's reliability dynamics observation in the space of parameters connected with a failure. Using parameters correctness distribution in the form of fuzzy sets membership function, and a chaos generator in the form of logistic map we obtained the bifurcation diagrams of the system reliability functioning with redundancy of the elements.

It is shown that increasing the number of redundant elements postpones the moment of the first bifurcation connected with a loss of reliability, and decreases the orbit size around which there is an oscillation of the level of system's membership to the reliable state.

It was proposed the problem statement of redundancy optimization under chaotic oscillations of parameters, and the example of its solution by genetic algorithm is provided.

The complex systems reliability dynamics modelling by integration of fuzzy logic and chaos theory can be considered as a perspective direction of the future research.

\section{Appendix}

Genetic algorithms are well-known methodology for the reliability optimization problem solving [21]. It is based on a definition and coding of initial population of the solutions and application to them the operations of crossover, mutation, and selection according to the given fitness function. The convenient tool of application of this methodology is MATLAB which provide the automation of coding and basic operation. To apply the MATLAB tools we need to define the fitness function.

$P=\left\{\mathbf{Y}^{j} \mid j=1,2, \ldots, L\right\}$ is a population, where $\mathbf{Y}^{j}=$ $\left(y_{1}^{j}, y_{2}^{j}, \ldots, y_{n}^{j}\right)$ is a $j$ th chromosome solution, and $L$ is a size of population.

Operations of crossover and mutation were implemented in MATLAB. Mutation coefficient was chosen experimentally and was equal to 0.1 . Fitness function $\mathrm{FF}\left(\mathbf{Y}^{j}\right)$ was chosen with the respect to the total expenses for the redundancy (5) and penalty function $\operatorname{PF}\left(\mathbf{Y}^{j}\right)$ as follows:

$$
\mathrm{FF}\left(\mathbf{Y}^{j}\right)=\sum_{i=1}^{n} c_{i} \cdot y_{i}^{j}+\operatorname{PF}\left(\mathbf{Y}^{j}\right)
$$

The use of penalty function allows eliminating solutions, which do not satisfy condition (8), by assigning them the value (1000), which is much greater than first member of summation in (A.1). This number 1000 was chosen experimentally. So we have

$$
\operatorname{PF}\left(\mathbf{Y}^{j}\right)= \begin{cases}0, & \text { if condition }(8) \text { is satisfied } \\ 1000, & \text { otherwise }\end{cases}
$$

As an example, Figure 12 shows the results redundancy optimization by genetic algorithm in MATLAB when $\mu_{B}^{*}=$ 0.8 and $a^{*}=2.9$.

The upper part of the Figure 12 shows the dynamics of fitness function changing with the growth of the generations number. The lower part shows the current optimal solution $\mathbf{Y}=(2,1,0,1,2)$. It took 300 generations to find the optimal solution with population size of $L=250$.

\section{References}

[1] B. V. Gnedenko, Y. K. Belyaev, and A. D. Solovyev, Mathematical. Methods of Reliability, Theory, Academic Press, New York. NY, USA, 1969.

[2] R. E. Barlow and F. Proshan, Mathematical Theory of Reliability, Wiley, London, UK, 1965.

[3] L. Zadeh, "The concept of a linguistic variable and its application to approximate reasoning-I-III," Information Sciences, vol. 8, no. 3, pp. 199-251, 1975, no. 4, pp. 301-357, 1975-no. 9, pp 43-80, 1976.

[4] L. A. Zadeh, "Fuzzy sets as a basis for a theory of possibility," Fuzzy Sets and Systems, vol. 1, no. 1, pp. 3-28, 1978.

[5] G. E. P. Box, G. M. Jenkins, and G. C. Reinsel, Time Series Analysis: Forecasting and Control, Prentice Hall, Englewood Cliffs, NJ, USA, 3rd edition, 1994.

[6] J. C. Sprott, Chaos and Time-Series Analysis, Oxford University Press, 2003.

[7] N. V. Butenin, Y. I. Nejmark, and N. A. Fufaev, An Introduction to the Theory of Nonlinear Oscillations, Nauka, Moscow, Russia, 1987.

[8] "Reliability and safety analysis under fuzziness," in Studies in Fuzzyness, T. Onisawa and J. Kasprzyk, Eds., vol. 4, Phisika, A Springer Company, 1995.

[9] K. Y. Cai, Introduction on Fuzzy Reliability, Kluwer Academic Publishers, Boston, Mass, USA, 1996.

[10] A. Rotshtein and S. Shtovba, Fuzzy Reliability Analysis of Algorithmic Processes, Continent-PRIM, Vinnitsa, Ukraine, 1997.

[11] L. Utkin and I. Shubinsky, Nontraditional Methods of the Informational Systems. Reliability Estimation, Lubavich Publishing House, St. Petersburg, Russia, 2000.

[12] A. Rotshtein, "Fuzzy reliability analyses of human's algorithms activity," Reliability, vol. 21, no. 2, pp. 3-18, 2007 (Russian).

[13] A. Rotshtein, "Fuzzy-algorithmic analysis of complex systems reliability," Cybernetics and Systems Analysis, vol. 47, no. 6, pp. 919-931, 2011.

[14] V. M. Glushkov, "Automata theory and formal microprogram transformations," Cybernetics, vol. 1, no. 5, pp. 1-8, 1965 (Russian).

[15] D. I. Katelnikov and A. P. Rotshtein, "Fuzzy algorithmic simulation of reliability: control and correction resource optimization," Journal of Computer and Systems Sciences International, vol. 49, no. 6, pp. 967-971, 2010.

[16] W. J. Kolarik, J. C. Woldstad, S. Lu, and H. Lu, "Human performance reliability: on-line assessment using fuzzy logic," IIE Transactions, vol. 36, no. 5, pp. 457-467, 2004.

[17] F. Z. Zou and C. X. Li, "Chaotic model for software reliability," Chinese Journal of Computers, vol. 24, no. 3, pp. 281-291, 2001. 
[18] S. Dick, C. L. Bethel, and A. Kandel, "Software-reliability modeling: the case for deterministic behavior," IEEE Transactions on Systems, Man, and Cybernetics Part A, vol. 37, no. 1, pp. 106-119, 2007.

[19] R. M. May, "Simple mathematical models with very complicated dynamics," Nature, vol. 261, no. 5560, pp. 459-467, 1976.

[20] B. A. Kozlov and I. A. Ushakov, Handbook on Reliability of Radio and Automation Systems, Soviet Radio, Moscow, Russia, 1975.

[21] M. Gen and R. Cheng, Genetic Algorithms and Engineering Design, John Wiley \& Sons, 1997. 

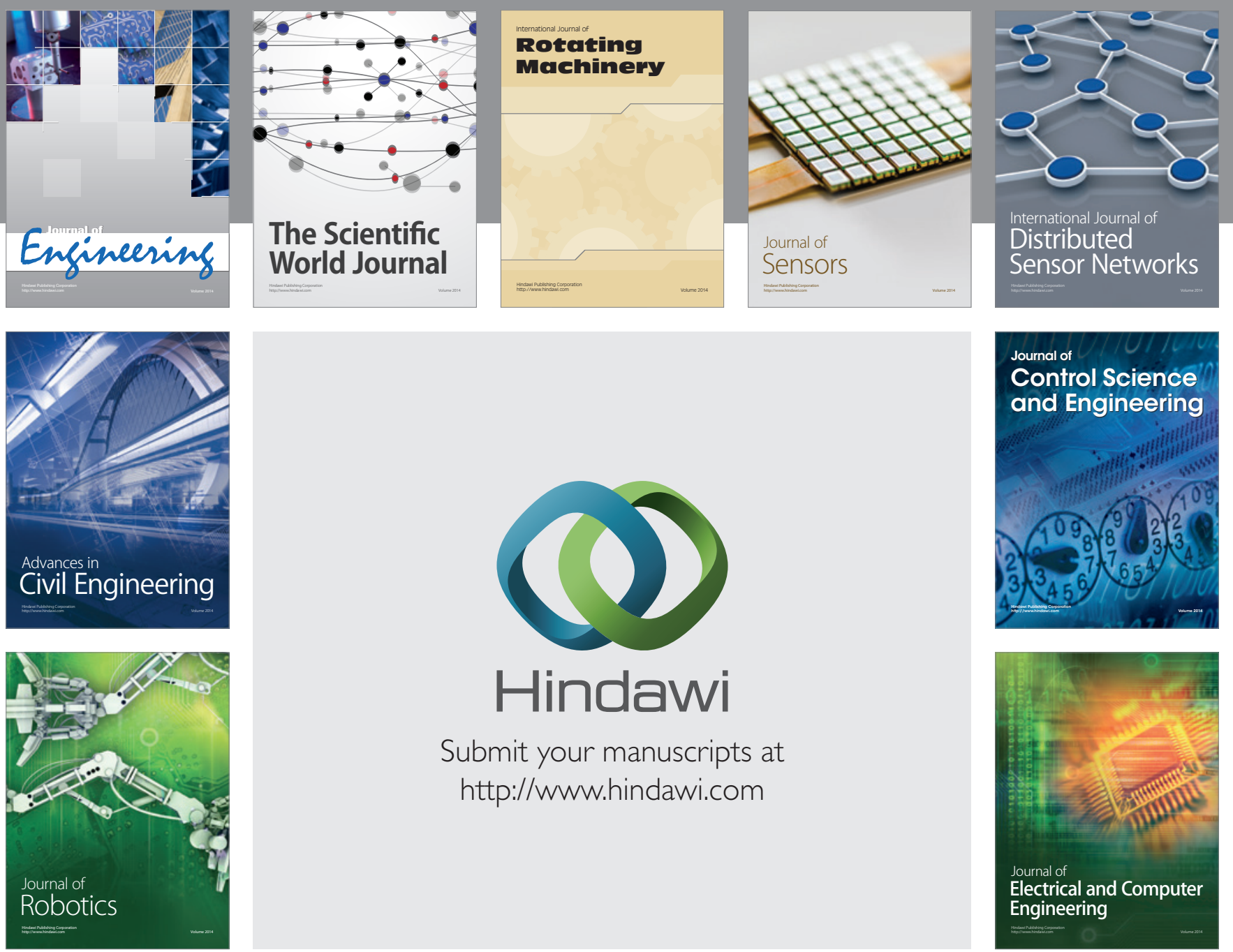

Submit your manuscripts at

http://www.hindawi.com
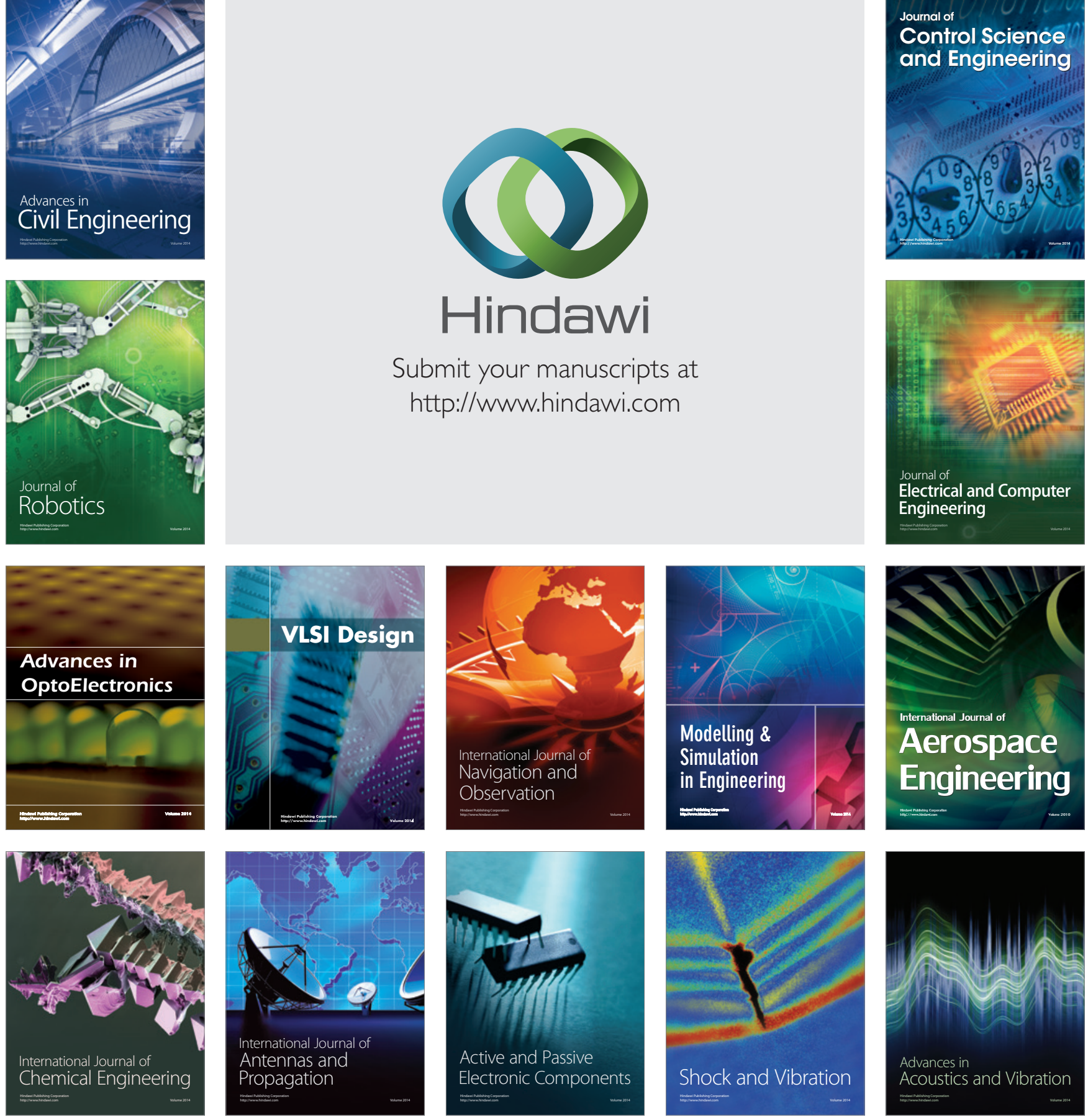